International Journal of Modern Physics A

(C) World Scientific Publishing Company

\title{
KERR-NEWMAN ELECTRON AS SPINNING SOLITON
}

\author{
ALEXANDER BURINSKII* \\ Theor.Phys. Lab., NSI, Russian Academy of Sciences, B. Tulskaya 52 Moscow, 115191 Russia. $^{\dagger}$ \\ first_author@domain_name
}

Received Day Month Year

Revised Day Month Year

\begin{abstract}
Measurable parameters of the electron indicate that its background should be described by the Kerr-Newman (KN) solution. Spin/mass ratio of the electron is extreme large, and the black hole horizons disappear, opening a topological defect of spacetime - the Kerr singular ring of the Compton size, which may be interpreted as a closed fundamental string to the low energy string theory. The singular and twosheeted structure of the corresponding Kerr space has to be regularized, and we consider the old problem of regular source of the KN solution. As a development of the earlier Keres-Israel-HamityLópez model, we describe the model of smooth and regular source forming a gravitating and relativistically rotating soliton based on the chiral field model and the Higgs mechanism of broken symmetry. The model reveals some new remarkable properties: 1) the soliton forms a relativistically rotating bubble of the Compton radius, which is filled by the oscillating Higgs field in pseudo-vacuum state, 2) boundary of the bubble forms a domain wall which interpolates between the internal flat background and the external exact Kerr-Newman (KN) solution, 3) phase transition is provided by a system of the chiral fields, 4) vector potential of the external the KN solution forms a closed Wilson loop which is quantized, giving rise to quantum spin of the soliton. 5) soliton is bordered by a closed string, which is a part of the general complex stringy structure.
\end{abstract}

Keywords: Kerr's gravity; electron; soliton; $\mathrm{N}=2$ string.

PACS numbers:

\section{Introduction and summary}

It is now commonly accepted that black holes $(\mathrm{BH})$ have to be associated with elementary particles. Physics of black holes is based on the complex analyticity, which unites them with quantum and superstring theories and particle physics.

In spite of these evident relationships, Gravity and Quantum theory are conflicting and cannot be unified in a whole theory. Similarly, the path from Superstring theory to particle physics represents also a still unsolved problem, and as it was recently claimed by John Schwarz, “... realistic model of elementary particles still appears to be a distant dream..." .

$\dagger$ e-mail: bur@ibrae.ac.ru. 
Principal point of the conflict between gravity and quantum theory is related with the statement on the pointlike and structureless quantum electron. This point cannot be accepted by gravity, which requires an extended soliton-like structure of the electron, as a field distribution with a regular energy-momentum tensor in configurational space. Contrary, quantum theory suggests the claim on the pointlike structure of the electron, or its statistic description by a wave function. String theory replaced the point-like quantum particles by the extended strings and membranelike sources. However, the principal quantum particle - electron, is still considered as point-like. In particular, Frank Wilczek writes in!2 "...There's no evidence that electrons have internal structure (and a lot of evidence against it)". Similarly, the superstring theorist Leonard Susskind notes that electron radius is "...most probably not much bigger and not much smaller than the Planck length..", ${ }^{3}$ It should be mentioned that this point of view is supported by the high energy scattering, which have not found the electron structure down to $10^{-16} \mathrm{~cm}$. However note, Quantum Electrodynamic considers an effective size of a "dressed electron", which corresponds to the Compton region of vacuum polarization. Although space-time structure of this region is usually not discussed, some hint is coming from the relativistic quantum mechanics, which indicates zitterbewegung of the electron, a lightlike helicoidal motion following from the Dirac equation.

These partial indications on the peculiar role of the Compton zone of the electron find unexpectedly strong support from Kerr's gravity.

It was obtained by Carter that the Kerr-Newman (KN) solution, which is exact solution of the Einstein-Maxwell gravity for a charged and rotating black-hole (BH), has the gyromagnetic ration $g=2$ as that of the Dirac electron. Therefore, the four experimentally observable parameters of the electron: spin $J$, mass $m$, charge $e$ and magnetic moment $\mu$ indicate unambiguously that gravitational background of the electron should be described by the KN solution. Extremely large spin of the electron with respect to its mass should produce an over-rotating Kerr geometry without horizon, which displays a naked topological defect of space-time in the form of the "Kerr singular ring" of the radius $a=\hbar / 2 m$, which is half of the Compton wave length $\lambda_{c}=\hbar / m$. This singular ring turns out to be branch line of the space into two sheets resulting in a two-fold structure of the electron background. The corresponding gravitational and electromagnetic fields of the electron are concentrated near the Kerr ring, forming a sort of a closed string, structure of which turns out to be close to the described by Sen heterotic string solution. 5 This contradicts to the statements on the structureless electron and is very far from the its Planck size suggested by superstring theory. However, it confirms the peculiar role of the Compton zone of the "dressed" electron of Quantum electrodynamics, matches with the known limit of the localization of the Dirac electron ${ }^{6}$ and indicate relationships with string theory.

There appear two questions:

(A) How does the KN gravity know about one of the principal parameters of Quantum theory? and 
(B) Why does Quantum theory works successfully on the flat spacetime, ignoring such strong defect of the background geometry?

A small and slowly varying gravitational field could be neglected, however the stringlike KN singularity forms a branch-line of the KS spacetime, and such a topological defect cannot be ignored. A natural resolution of this trouble could be the assumption that there is an underlying structure, or even the theory providing the consistency of quantum theory and gravity. We conjecture that such underlying structure is to be the Kerr geometry, complex structure of which indicates close relations to a four-dimensional version of superstring theory, the "mysterious" $\mathrm{N}=2$ superstring theory which is consistent in four dimensions, but does not have the standard string interpretation.$^{7}$ It has been suggested in ${ }^{8}$ that it may be considered as an alternative to the higher dimensional superstring theory.

In this paper, we consider structure of the real source of the Kerr geometry. Starting in sec.2 from motivations to consider the Kerr singular ring as a closed heterotic string, we discuss in sec. 3 peculiarities of the over-rotating Kerr geometry (without horizons), singular ring, twosheetedness and specifical properties of the Kerr coordinate system. In sec.4 we we consider development the models of source of the $\mathrm{KN}$ solution, and in sec.5 consider regularization of the $\mathrm{KN}$ solution by the Higgs fields, which creates the source of the KN solution as a gravitating soliton in the form of a rotating superconducting disk of the Compton radius with a closed relativistic string situating at the disk perimeter. In this section we obtain some remarkable peculiarities of the spinning soliton model:

a) oscillations of the Higgs field with the frequency $\omega=2 m$, where $m$ is mass of the soliton,

b) appearance of the quantum loop of the vector potential (Aharonov-BohmWilson loop), which is wrapped around the disk-like source providing quantization of the soliton spin.

In sec.6 may be the most hard for the reading, since we consider there the field aspect of this model, and show that for consistency with gravity we need to extend the Higgs field model to chiral model containing a triplet of the chiral fields. This complication provides a phase transition from the external exact $\mathrm{KN}$ solution to a regular source, interior of with is build of pseudovacuum state of the Higgs field resulting in flat metric in the disk-like core of the KN solution. Therefore we obtain the desirable flat background in vicinity of the $\mathrm{KN}$ source, answering the second above-mentioned question. Finally, in conclusion we discuss some relations of this model with QED and superstring theory.

\section{Kerr singular ring as a string, first qualitative treatment}

The Kerr singular ring is generated as a caustic of Kerr congruence or the focusing line. The KN gravity indicates that this string should represent one of the principal elements of the extended electron structure.

The widespread opinion that the range of interaction for gravitational field is 
"tremendously weak and becomes compatible to other forces only at Planck scale" is inspired by the usual analysis of the spherically symmetric Schwarzschild solution, which has a characteristic radius of the interaction (determined by the position of horizon, $r_{g}=2 m$ ) proportional to the mass parameter. The Kerr geometry breaks this predicate, and moreover, it turns this dependence into inverse one, $r_{g} \sim$ $J / m$, showing that the area of expansion of the Kerr gravitational field is inversely proportional to mass and proportional to spin of the system. This unexpected effect follows from the Kerr relation for the radius of the Kerr singular ring, $a=J / m$, which shows that a strong gravitational field may occupy very large region for the objects of low masses $m$ and large angular momentum $J$, which is just the case corresponding to elementary particles. This seeming paradox has very simple explanation - gravitational field of the Kerr solution vanishes at the centrum of the solution and concentrates in a thin vicinity of the Kerr singular ring, forming a type of "gravitational waveguide", or string for propagation of circular waves, as it was suggested more than forty years ago in. ${ }^{9}$ Although, this simple model of the electron is very naive, it gives intuitive explanation to many important facts:

(i) first of all we obtained that the Kerr singular ring represented a waveguide for propagation of circular waves, which corresponded to circular motion of a massless particle. In the modern terms of the dual string model, there appeared a type of the four-dimensional "compactification without compactification",

(ii) mass of the particles originated as energy of the massless excitations, which is similar to origin of the mass spectrum in string theory and corresponded to the old Wheeler model of the "mass without mass",

(iii) the process of mutual transformation of the massive and massless particles, in particular, annihilation of the electron positron pair had got natural intuitive explanation,

(iv) circular motion of the photon with the wave-length $\lambda$ and the energy $E=h c / \lambda$ created relativistic increase of mass $m=m_{0} / \sqrt{1+(v / c)^{2}}$, which followed from the simple geometric relations,

(v) there appeared natural explanation of the "zitterbewegung" of the Dirac electron,

(vi) quantum spin could be interpreted as a consequence of the Bohr quantization of the photon waves wrapped around the Kerr ring,

(vii) the half-integer wave-lengths corresponding to half-integer spin and the spinor twosheetedness could be related with twosheeted structure of the Kerr spacetime,

(viii) the wave-particle dualism and origin of the de Broglie waves have got natural explanation.

It was too much for such a simple model. Of course, there appeared also problems. Principal trouble of this model is the question: What could keep the photon on the orbit of the Compton radius? Estimations of the Schwarzschild gravitational field showed that it is too weak at the Compton distances. However, contrary to the 
Schwarzschild solution, which has a 'range' of the gravitational field proportional to the mass of the source (radius of the horizon $r_{g}=2 m$ ) a, the new dimensional parameter of the KN geometry $a=J / m$, which grows with angular momentum $J$ and has the reverse mass-dependence. As a result, the zone of gravitational interaction determined by parameter $a$ increases for the large angular momentum and small masses, and therefore, it turns out to be essential for elementary particles. The reason of that is a specific structure of the KN gravitational field, which concentrates near the Kerr singular ring, forming a closed gravitational waveguide - a type of the closed gravitational string. [

Kerr-Newman (KN) solution has gyromagnetic ratio $g=2$, as that of the Dirac electron, ${ }^{4}$ and therefore, at least the asymptotic gravitational and electromagnetic (em) field of the electron should correspond to the KN solution with great precision. Because of that, the charged Kerr-Newman $(\mathrm{KN})$ solution ${ }^{10}$ has paid attention as a classical background of electron,,$[11,20$

\section{Over-rotating Kerr geometry and twosheetedness}

The spin/mass ratio of the elementary particles $a=J / m$ is extremely high. In the dimensionless units $c=G=\hbar=1$, it is about $10^{22}$, while already for $a / m>1$ the $\mathrm{BH}$ horizons disappear. It indicates that spinning particles should correspond to over-rotating BH solutions, for which the BH horizons disappear, and there appears a naked Kerr singular ring. The electron background acquires a source in the form of a closed string of the Compton radius $a=\hbar / 2 m$.

The over-rotating KN has simple representation in the Kerr-Schild (KS) form of metric, 11

$$
g_{\mu \nu}=\eta_{\mu \nu}+2 H k_{\mu} k_{\nu}
$$

where $\eta_{\mu \nu}$ is metric of an auxiliary Minkowski background in Cartesian coordinates $\mathrm{x}=x^{\mu}=(t, x, y, z), H=H(x)$ is a scalar function, and $k^{\mu}=(1, \vec{k})$ is a null 4-vector field. Using signature $(-+++)$ we obtain $k_{\mu} k^{\mu}=(\vec{k})^{2}-1=0$, and consequently $\vec{k}$ is a unit spacelike vector field, $(\vec{k})^{2}=1$. The use of auxiliary Minkowski background allows to avoid dependence of the metric on position of the horizon. The KS metric form is extreme simple, and one wonders how it can describe the Kerr metric which is known as very complicate. The reason is hidden in the very complicate form of the vector field $\vec{k}$, which represents a vortex of the so-called Pricipal Null Congruence (PNC), or simple Kerr congruence. The form of field $\vec{k}(x)$ is shown in Fig.1.

Four vector $k_{\mu}(\mathrm{x})$, tangent direction to PNC, represents a family of the lightlike lines, which form a skeleton of the Kerr geometry. These lines are twistors. The reader should not be frightened by word 'twistor'. Indeed, twistor theory is excessively mathematized, but physical meaning of a twistor is very simple: it is a geodesic line of a photon (lightlike or null line) passing aside of the coordinate origin

${ }^{\mathrm{a}}$ We use the natural units $\hbar=G=c=1$, in which $e^{2}=\alpha \approx 137^{-1}$. 


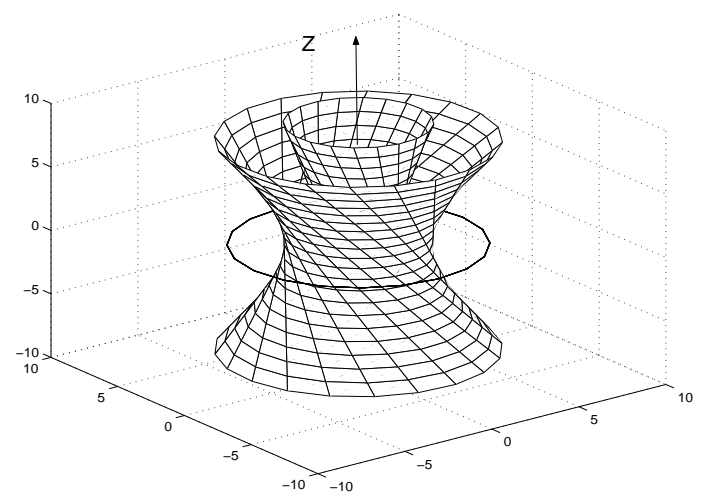

Fig. 1. Twistor null lines of the Kerr congruence are focusing on the Kerr singular ring, which forms a branch line of space in two sheets.

or position of the observer. Twistorial Kerr congruence means that each point of the Kerr spacetime is polarized, and has a selected lightlike direction. All the tensor fields of the Kerr geometry turns out to be aligned with this selected lightlike direction.

The factor $H$ in (1) is a scalar function, which for the charged Kerr-Newman $(\mathrm{KN})$ solution takes the form

$$
H=\frac{m r-e^{2} / 2}{r^{2}+a^{2} \cos ^{2} \theta},
$$

where $r=0$ and $\theta=0$ are oblate spheroidal coordinates which are adapted to twisted and twosheeted structure of the Kerr congruence. The KS formalism uses a few different coordinate systems, which allows one to adapt treatment to different aspects of the Kerr solution and simplify calculations. The oblate spheroidal coordinates represent a family of oblate ellipsoids $r=$ const. and confocal family of the hyperboloids $\theta=$ const., see Fig.2. The Kerr oblate spheroidal coordinates $r$ and $\theta$ and $\phi_{K}$ are related with minkowskian coordinates as follows,

$$
x+i y=(r+i a) e^{i \phi_{K}} \sin \theta, \quad z=r \cos \theta, \quad \rho=t-r,
$$

where $\rho$ is the additional 'light cone'or 'retarded time' coordinate, which describes propagations of the waves along the null rays of the Kerr congruence. However, it will not be essential for our treatment here.

The naked Kerr singular ring which corresponds to $r=0$ and $\theta=\pi / 2$. It is a focus line of the null directions $k^{\mu}$, and simultaneously, it is a branch line of the Kerr space into two sheets, $r<0$, and $r>0$. The oblate spheroidal coordinates are twosheeted and adapted to two sheets of the Kerr geometry. One sees that if congruence is in-going on the sheet $r<0$, its analytic extension is to be outgoing on the sheet $r>0$. The null directions $k_{\mu}$, and metric (1) turn out to be different on the 'positive' and 'negative' sheets. We obtain that the background is spoiled at the Compton distances $a=\hbar / 2 m$, getting a strong topological defect 


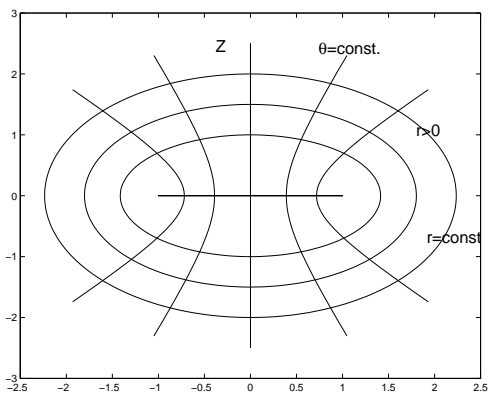

Fig. 2. Oblate coordinate system $r, \theta$ with focal points at $r=\cos \theta=0$ covers the space-time twice, for $r>0$ and $r<0$.

in the form of the Kerr singular ring and twosheetedness. This twosheeted space is reminiscent of the old Einstein-Rosen bridge, or the discussed later Wheeler's worm-hole, which allows one to create a 'charge without charge' preventing the space-time from formation of the singularities. Strong curvature of the background in the Compton region indicates that a regularization, analogous to regularization of charge, has to be executed for the metric too. In fact, this strong breakdown of space indicates that the Compton region has to be a zone of new physics. In accord to results of Quantum Electrodynamics this zone should be related with processes of vacuum polarization.

\section{The 'bubble' and string-like sources of $\mathrm{KN}$ solution.}

Bubble source. It is remarkable, that the Kerr geometry gives unambiguous answer on the size and shape of this zone, 14 One sees that the KS metric (11) becomes flat for the vanishing function $H$, and therefore, one should set $H=0$ for regularization of the metric in the source of KN solution. The boundary of this source is determined from (2) by the value of the Kerr ellipsoidal radial coordinate

$$
r=r_{e}=e^{2} /(2 m),
$$

inside of which, $r<r_{e}$, the space-time should be set as flat, $H=0$. We obtain that the source of KN solution should represent an ellipsoidal shell with flat interior which resembles a vacuum bubble. The bubble has form on a highly oblate disk. One sees from the coordinate relations (3), that for $r=r_{e}$, radius of the disk (corresponding to $z=0$ ) is $\sqrt{x^{2}+y^{2}}=\sqrt{r_{e}^{2}+a^{2}}$, while the disk thickness (corresponding to $x^{2}+$ $y^{2}=0$ ) is determined by $z_{\text {disk }}=r_{e}$, and therefore, the ratio thickness/radius turns out to be $r_{e} / \sqrt{r_{e}^{2}+a^{2}}$. In the natural units $(\hbar=G=c=1) e^{2} \approx 137^{-1}$, and $r_{e}=$ $e^{2} /(2 m) \approx 137^{-1} /(2 m)$. From the Kerr relation $J=m a=\hbar / 2=1 / 2$, we have $a=$ $1 /(2 m)$, and therefore $r_{e}=a / 137<<a$. We obtain that the ratio thickness/radius is close to $r_{e} / a=137^{-1}$, and determined by the fine structure constant $\alpha=137^{-1}$. The fine structure constant acquires in Kerr's electron a geometric meaning! A very important specification was given by Hamity, $\stackrel{23}{2}$ who noticed that the disk should 
be rigidly rotating reaching the velocity of the light at the edge border.

Let's look now at the electromagnetic (EM) field. The vector potential of the $\mathrm{KN}$ solution is given by

$$
A_{K N}^{\mu}=R e \frac{e}{r+i a \cos \theta} k^{\mu}
$$

One sees that it is also proportional to the null direction $k^{\mu}$, and therefore, it is also aligned with the Kerr congruence (PNC), i.e.

$$
A_{K N}^{\mu} g_{\mu \nu} k^{\nu}=0
$$

This is principal property of the algebraically special solutions, that all the tensor quantities are aligned with PNC, which simplifies solutions of the field equations.

This source was suggested by López as a classical model of an extended electron in general relativity ${ }^{14}$ Like the other shell-like models, the López model was not able to explain the origin of Poincaré stress, and the necessary tangential stress was introduced by him phenomenologically, as a distribution over the surface of the rotating shell.

Meanwhile, the necessary tangential stress appears naturally in the field models of domain walls. The corresponding field model of the domain wall bubble was suggested in $24 \sqrt[25]{25}$ and developed in $\frac{17}{17}$ as a gravitating soliton model, in which the Higgs field is concentrated inside the bubble in a pseudo-vacuum superconducting state and performs regularization of the KN solution.

The Higgs field pushes the em field from the bubble. As a result, the em field is regularized, acquiring the cut-off parameter $r_{e}$ leading to maximal value of the vector potential

$$
A_{\text {max }}^{\mu}=\operatorname{Re} \frac{e}{r_{e}} k^{\mu}=\operatorname{Re} \frac{2 m}{e} k^{\mu}
$$

which is reached on the boundary of the disk corresponding to $\cos \theta=0$.

The regularized KN space tends to flat near the source, in agreement with the requirements of Quantum theory. However, it should be noted, that performing regularization we distorted the original KN solution. Submission of the regular EM field and metric in the Einstein-Maxwell system of the field equations will produce the additional charge, currents and matter on the surface of the bubble in the form of a density distributions described by $\delta$-function 14 These tensor densities take a simple diagonal form in a corotating system of coordinates, which evidences that the disk-like bubble has to be rigidly rotating, and the linear velocity at the boundary of the disk is to be close to the velocity of light. In the recent development, this model takes the form of a gravitating soliton, $\frac{17}{17}$ in which the infinite thin shell of this bubble is replaced by a domain wall boundary, and the bubble is not empty, but is filled by the Higgs field in a pseudovacuum state. A special set of the chiral fields performs a phase transition from the external exact KN solution to the Higgs field inside the bubble providing the flat internal metric and pseudovacuum state of the Higgs field. 
String-like source. Let's now consider stringy interpretation of the Kerr singular ring, which was initially considered as an alternative model of the source of Kerr geometry. It was suggested in $\underline{9}$ to consider the Kerr ring as a closed relativistic string similar to strings of the dual models. The massless equations of the relativistic strings create the massive states from energy of string excitations. This mechanism is similar to the Wheeler's idea of 'geon', gravitational-electromagnetic object with 'mass without mass'. Mass of the 'geon' is generated by energy of the electromagnetic field, in particular, by photons traveling on the circular orbits. It was suggested in ${ }^{9}$ that the Kerr singular ring may represent a type of gravitational waveguide which keeps the electromagnetic $(\mathrm{em})$ or spinor waves in the orbital motion,, $9[12] 19$ Twenty years later, the string-like solitonic solutions with traveling waves were considered as fundamental strings solutions to low energy string theory. ${ }^{26}\left[27\right.$ It was been shown in ${ }^{28}$ that the field structure of the Kerr singular ring is similar to the structure of the fundamental heterotic string in the obtained by Sen solutions to low-energy string theory ${ }^{\sqrt{521}}$ Later on, it was obtained that this string is only "a tip of the iceberg", and a wonderful stringy system related with $\mathrm{N}=2$ superstring theory is indeed hidden in the complex Kerr geometry.

\section{Regularization of the em field by the Higgs fields.}

\subsection{Basic field equations.}

Regularization of the em field in the bubble-source model is performed by the Higgs mechanism of broken symmetry, which was used in many particle-like models, like the t'Hooft-Polyakov models and the the Nielsen-Oleser ${ }^{29}$ field model for the stringlike solution in superconductivity.

The em field in vacuum and in the Einstein-Maxwell theory is massless and long-distant. Presence of the Higgs field gives a mass to the em field making it short-distant. The em field cannot deeply penetrate in the regions occupied by the Higgs field. The depth of penetration $\delta \sim 1 / m$ depends on the acquired mass $m$. Because of that the Higgs field is used for description of the Meissner effect, interaction of the em field with ideal conductors and superconductors. There is also an opposite influence: the strong em field expels the Higgs field. For example, it penetrates in a superconductor in the form of vortex filaments, as it was described by Abrikosov solutions. The Higgs field model was used in the t'Hooft and Polyakov particle-like models of the magnetic monopole, and also was used by Nielsen and Olesen as a model of the dual relativistic string in a superconducting media. In particular, the Lagrangian used in $\frac{29}{}$ for the complex Higgs field $\Phi(x)$ interacting with the em vector field $A^{\mu}$ has the form

$$
\mathcal{L}_{N O}=-\frac{1}{4} F_{\mu \nu} F^{\mu \nu}+\frac{1}{2}\left(\mathcal{D}_{\mu} \Phi\right)\left(\mathcal{D}^{\mu} \Phi\right)^{*}+V(|\Phi|),
$$

where $\mathcal{D}_{\mu}=\nabla_{\mu}+i e A_{\mu}$ are to be covariant derivatives, and $F_{\mu \nu}=A_{\mu, \nu}-A_{\nu, \mu}$. We can use the similar field model. In the vicinity of the KN source we have $H \approx 0$ leading to flat space-time, which allows us to consider $\nabla_{\mu}$ as flat derivatives and 
to set $\nabla_{\nu} \nabla^{\nu}=\square$. This Lagrangian gives rise to the system of equations for the coupled Maxwell-Higgs system

$$
\begin{aligned}
\mathcal{D}_{\nu}^{(1)} \mathcal{D}^{(1) \nu} \Phi & =\partial_{\Phi^{*}} V, \\
\square A_{\mu}=I_{\mu} & =e|\Phi|^{2}\left(\chi,,_{\mu}+e A_{\mu}\right) .
\end{aligned}
$$

This system of the coupled equations describes an interplay of the Higgs and Maxwell field separated by some contact boundary and their mutual penetration through this boundary. Exact solutions of this system are known only for some particular cases of the potential $V$ and only for flat boundary. Analysis of the solutions requires usually numerical calculations or strong simplifications. It should also be noticed that the most of the known solutions to the Maxwell-Higgs system describe the localized em field confined in a restricted region of space surrounded by the Higgs field, i.e. a cloud of the gauge massless fields surrounded by a superconducting media formed by Higgs field. Meanwhile, our task is to consider opposite situation, in which the Higgs field is localized inside the bubble and surrounded by the massless em field extended to infinity. At first sight, this difference seems inessential, but indeed, our case of interest becomes much more complicated and requires introduction of the several Higgs-like fields and the potential $V$ of an especial domain wall form. We will discuss resolution of this problem in the final section of our treatment, but now we consider some extra simplifications, which will allow us to clarify basic peculiarities of the regular source of the $\mathrm{KN}$ solution.

\subsection{The source without rotation.}

We can further simplify the problem considering the source without rotation. By setting $a=0$, we obtain that the Kerr singular ring shrinks to a point and the vector potential (5) takes the spherically symmetric form

$$
A_{0}^{\mu}=\frac{e}{r} k^{\mu},
$$

where $r$ is the usual real radial coordinate, $r=\sqrt{x^{2}+y^{2}+z^{2}}$. The null vector field $k^{\mu}$ turns into a spherically symmetric system of the four-vectors $k^{\mu}=(1, \vec{n})$, where $\vec{n}=x^{\mu} / r$ represents a hedgehog of the unit radial directions. The metric (??) and vector potential (11) correspond to the Reissner-Nordström solution in the Kerr-Schild form.

The bubble-source filled by Higgs field takes in this case the spherical form, and the maximal value of regularized vector potential will be again

$$
A_{\text {max }}^{\mu}=\operatorname{Re} \frac{e}{r_{e}} k^{\mu}=R e \frac{2 m}{e} k^{\mu},
$$

which corresponds to the classical model of the electron as a charged sphere with a unique difference that the sphere is replaced by a superconducting ball, interior of which is filled by a complex Higgs field $\Phi(x)=\Phi_{0} \exp \{i \chi(x)\}$, where $\Phi_{0}$ is the vacuum vacuum expectation value ('vev') of the Higgs field. 
Therefore, we replace the equation (9) by assumption on the sharp boundary of the bubble, setting $\Phi(x)=0$ outside the bubble and $\Phi(x)=\Phi_{0}$, for $r<r_{e}$. The corresponding em field is massless and has the form (5) for $r>r_{e}$, while penetrating inside the bubble it should satisfy the eq.

$$
\square A_{\mu}=I_{\mu}=e|\Phi|^{2}\left(\chi, \mu+e A_{\mu}\right) .
$$

If the phase $\chi$ is a constant, this equation turns into $\square A_{\mu}=m_{v}^{2} A_{\mu}$, where $m_{v}=|e \Phi|$ is the mass acquired by vector field $A_{\mu}$ due to Higgs mechanism. Here we have another action of the Higgs field, which is related with compensating role of the phase $\chi$ representing a space-time function $\chi(x)$. Splitting $A_{\mu}=\left(A_{0}, \vec{A}\right)$ into timelike component $A_{0}=\frac{e}{r}$ and the radial field $\vec{A}=\frac{e}{r} \vec{n} \equiv e d \ln r$, one can drop formally the radial part as a full differential, which will not products the strengths of the em field $F_{\mu \nu}$. In accord to (13), the time-like component $A_{0}=\frac{e}{r}$ could create the charge

$$
\rho=I_{0}=e|\Phi|^{2}\left(\chi, 0+e A_{0}\right)
$$

However, the charge and current have to be expelled from superconducting interior of the bubble to its boundary. It sets the condition $I_{0}=0$ for $r<r_{e}$ resulting in the the relation $\chi_{, 0}+e A_{0}^{i n}=0$, which fixes the value of component $A_{0}$ inside the bubble as a constant determined by the frequency of oscillations of the Higgs field $\omega$

$$
A_{0}^{(i n)}=-\frac{1}{e} \partial_{t} \chi=-\frac{1}{e} \omega .
$$

Note, that the left side of the (13), $\square A_{0}^{(i n)}=0$, is satisfied by the constant value of $A_{0}^{(i n)}$. At the boundary of the bubble $r_{e}$, the constant value $A_{0}^{(i n)}$ has to be matched with external solution $A_{0}=\frac{e}{r}$,

$$
A_{0}^{(i n)}=-\omega / e=\frac{e}{r_{e}} .
$$

Using $r_{e}=e^{2} /(2 m)$, we arrive at very important result that the Higgs field forms a coherent vacuum state oscillating with the frequency

$$
|\omega|=2 m
$$

Solitonic solutions of this type were called "oscillons". Solutions of this type were first considered by G.Rosen ${ }^{30}$ and Coleman ${ }^{31}$ (Q-ball). Examples of the oscillon solutions are spinning Q-balls $32[33$ and the bosonic star solutions. $[4$

\subsection{Inclusion of the rotation.}

Let us write now the Higgs phase in the form $\chi=\omega t+n \phi+\chi_{1}(r)$, where we have taken into account the constant frequency $\omega$, periodicity in $\phi$ and some dependence on $r$. The Higgs field inside the bubble can be represented as follows

$$
\Phi=|\Phi| \exp \left\{i\left(\omega t+n \phi+\chi_{1}(r)\right)\right\}
$$


where the azimuthal coordinate $\phi$ my be expressed via cartesian coordinates as follows $\phi=-i \ln [(x+i y) / \rho], \rho=\left(x^{2}+y^{2}\right)^{1 / 2}$.

In the rotating case the $\mathrm{KN}$ gauge field $A_{\mu}$, given by (5), is twisted, since it is aligned with tangent vector to twisted Kerr congruence, $k_{\mu}$. One sees, that the basic expressions for the Kerr metric (11) and the em field (5) are extreme simple. The complicated structure of the Kerr solution is concentrated in the form of vector field $k^{\mu}(x)$ which may be described in differential form in the Cartesian or the Kerr angular coordinates. The Cartesian representation is more important from theoretical point of view, since it shows relation to twistors, the Kerr theorem and superstrings ${ }^{[8}$ For our aims here, it is enough to give $k^{\mu}$ in differential form expressed in the Kerr angular coordinates ${ }^{11}$

$$
k_{\mu} d x^{\mu}=d r-d t-a \sin ^{2} \theta d \phi_{K} .
$$

The Kerr azimuthal coordinate $\phi_{K}$ has a very specific form determined by the relation (3). It is inconsistent with the standard angular coordinate $\phi$ of the Higgs field, and their differentials are related as follows

$$
d \phi_{K}=d \phi+\frac{a d r}{r^{2}+a^{2}}
$$

Fig. 4 shows that the spacelike part of $k^{\mu}=(1, \vec{k}), \vec{k}$ is tangent to Kerr ring in the equatorial plane at $r=0$. It means that the Kerr ring is lightlike, i.e. it slides along itself with the speed of light.

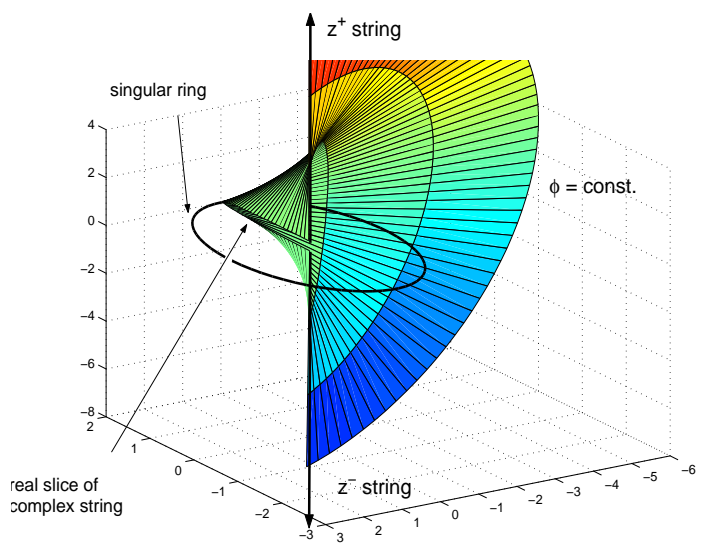

Fig. 3. The Kerr surface $\phi=$ const. The Kerr congruence is tangent to singular ring at $\theta=\pi / 2$.

The KN vector potential (5) takes the form

$$
A_{\mu} d x^{\mu}=\frac{-e r}{r^{2}+a^{2} \cos ^{2} \theta}\left[d r-d t-a \sin ^{2} \theta d \phi_{K}\right] .
$$

Using (20), one can express it in terms of $d \phi$ and obtain

$$
A_{\mu} d x^{\mu}=\frac{-e r}{r^{2}+a^{2} \cos ^{2} \theta}\left[d t+a \sin ^{2} \theta d \phi\right]+\frac{e r d r}{\left(r^{2}+a^{2}\right)},
$$


which shows that radial component $A_{r}$ represents a full differential and can be dropped $\mathrm{b}$ At the external side of the bubble boundary, $r=r_{e}+0=e^{2} / 2 m$, the the potential takes the value $A_{\mu}\left(r_{e}\right) d x^{\mu}=\frac{-e r_{e}}{r_{e}^{2}+a^{2} \cos ^{2} \theta}\left[d t+a \sin ^{2} \theta d \phi\right]$, and in the equatorial plane, $\cos \theta=0$, it forms the loop wrapped along the $\phi$-direction,

$$
A_{\mu}\left(r_{e}\right) d x^{\mu}=-\frac{2 m}{e}[d t+a d \phi],
$$

with tangent component $A_{\phi}\left(r_{e}\right)=-2 m a / e$. This field is penetrated inside the bubble,

$$
A_{\mu}^{(i n)} d x^{\mu}=-\frac{2 m}{e}[d t+a d \phi], .
$$

However, in agreement with (13), it is compensated by the phase of Higgs field, resulting in cancelling of the corresponding circular current inside the bubble,

$$
I_{\phi}^{(i n)}=0 \Rightarrow \chi,_{\phi}=-e A_{\phi}^{(i n)} .
$$

It implies periodic dependence of the Higgs field on $\phi, \Phi \sim \exp \{i n \phi\}$, with integer $n$. There appears the closed Wilson loop of the potential along the rim of disk-like source. The integral over the loop

$$
S=\oint e A_{\phi}\left(r_{e}\right) d \phi=-4 \pi m a
$$

has to be matched with periodic incursion of the Higgs phase $2 \pi n$, and therefore, it turns out to be quantized. Using the KN relation $J=m a$, we obtain wonderful result that the quantum Wilson loop of the $\mathrm{KN}$ em field $e \oint A_{\phi}^{(s t r)} d \phi=-4 \pi m a$ requires quantization of the total angular momentum of the soliton, $J=$ $m a=n / 2, n=1,2,3, \ldots$

Note, that this result together with the result related with Eq. (17) follow unambiguously from the form of KN solution and condition (4), and therefore, these results follow only from the requirement to get the KN source with a flat internal metric - from the requirement of consistency with flat background of quantum theory. This property was never observed in the other spinning Q-balls and the bosonic star solutions.

Extra note. By construction of the solution, the time-like and $\phi$ components of the obtained vector field are continuous at the boundary of the bubble $r_{e}$. However, there is discontinuity in their radial derivatives, which has to generate circular currents on the bubble boundary. Practically, the boundary is not sharp, and the vector field should have a finite depth of penetration $\delta_{v}$, and derivatives should be smoothed by this skin effect. The real values of the vector potential will differ from the obtained background solution (24) in the boundary layer $r \in\left[\begin{array}{ll}r_{e}, & r_{e}-\delta_{r}\end{array}\right]$. Denoting this deviation as $\delta A_{\mu}^{(\text {bound })}=A_{\mu}-A_{\mu}^{(i n)}$ in (13) we obtain the equation

$$
\square \delta A_{\mu}^{(\text {bound })}=I_{\mu}=e^{2}|\Phi|^{2} \delta A_{\mu}^{(\text {bound })}
$$

\footnotetext{
${ }^{\mathrm{b}}$ Inside the bubble $A_{r}$ is compensated by the $\chi_{1}(r)$ component of the Higgs phase.
} 
which shows that the em field acquires via Higgs mechanism the mass $m_{v}=e|\Phi|$, which produce the charge and ring-like current with the depth of penetration $\delta r \sim$ $1 / m_{v}$. It may be interpreted as a string-like massive and charged vector meson residing at the circular boundary of the bubble.

\section{Full action: potential, domain wall, gravity and phase transition.}

\subsection{Phase transition}

We have to consider now the role of the potential $V(\Phi)$ in formation of the boundary condition and in the phase transition from the external vacuum state corresponding to KN solution to some false-vacuum flat state inside the bubble source. In this point our model differs essentially from the other solitonic models, as well as from the known oscillon and Q-ball models. The renormalizable quartic potential $V=$ $\left(|P h i|^{2}-v\right)^{2}$, is generally used for the Higgs field models with broken symmetry. In particular, is was used by Graham for the oscillon model in ${ }^{33}$ and in the well known MIT and SLAC bag models. This is inappropriate for the gravitating soliton models, since presence of the Higgs field outside the source gives a mass to the external gravitational and EM fields turning them in the short-range fields. In particular, in our case it should distort the external KN field. Therefore, we have to use a different scheme of phase transition, in which the external vacuum state would be unbroken, and consequently, the Higgs field should be concentrated inside the source. This turns out to be a nontrivial task which cannot be solved by the typical quartic potential.

The similar problem appears in the Vilenkin-Witten model of the superconducting cosmic string,, 35 and to resolve this problem Witten used the $U(1) \times \tilde{U}(1)$ field mode ${ }^{\sqrt{35}}$ which contains two Higgs field: one of which $\Phi^{1}$ is concentrated inside the superconducting source, while another one $\Phi^{2}$ takes the complementary domain extended up to infinity. These two Higgs field are charged and adjoined to two different gauge fields $A^{1}$ and $A^{2}$, so that when one of them is long-distant in some region $\Omega$, the second one is short-distant in this region and vice-verse. This model is suitable for our case, however, in ${ }^{17}$ we used a supersymmetric scheme of phase transition ${ }^{36}$ suggested by Morris ${ }^{37}$ The Morris potential $V$ depends on two charged Higgs-like complex fields $\Phi$ and $\Sigma$ and one auxiliary uncharged real field $Z$, which are combined in the superpotential

$$
W=\lambda Z\left(\Sigma \bar{\Sigma}-\eta^{2}\right)+(Z+\mu) \Phi \bar{\Phi},
$$

where $\mu, \eta, \lambda$ are real constants. In accord to theory of the chiral superfields, $\frac{36}{36}$ the potential $V$ is determined from the superpotential $W$ by the relation

$$
V(r)=\sum_{i}\left|\partial_{i} W\right|^{2}
$$

where $\partial_{1}=\partial_{\Phi}, \partial_{2}=\partial_{Z}, \partial_{3}=\partial_{\Sigma}$, and $\Phi^{(i)}, i=1,2,3$ forms triplet of the chiral 
fields $c$

$$
\Phi^{(i)}=\{\Phi, Z, \Sigma\} .
$$

Vacuum states $V_{(v a c)}=0$ are determined by the conditions $\partial_{i} W=0$. It is easy to obtain for (28) two solutions:

(I) vacuum state: $Z=-\mu ; \Sigma=0 ;|\Phi|=\eta \sqrt{\lambda}$, corresponds to $W_{I}=\lambda \mu \eta^{2}$,

(II) vacuum state: $Z=0 ; \Phi=0 ; \Sigma=\eta$, corresponding to $W_{I I}=0$.

One can identify the field $\Phi$ as the main Higgs field, and the state (I), where $|\Phi|>0$, as the false-vacuum state of the Higgs field inside the bubble. Then the state (II), where $|\Phi|=0$, will be identified with external vacuum state with the non-zero Higgs-like field $|\Sigma|=\eta^{2}>0$.

The coupled with gravity action reads

$$
S=\int \sqrt{-g} R d^{4} x\left(\frac{R}{16 \pi G}+\mathcal{L}^{m a t}\right),
$$

where the full matter Lagrangian takes the form

$$
\mathcal{L}^{m a t}=-\frac{1}{4} F_{\mu \nu} F^{\mu \nu}+\frac{1}{2} \sum_{i}\left(\mathcal{D}_{\mu}^{(i)} \Phi^{(i)}\right)\left(\mathcal{D}^{(i) \mu} \Phi^{(i)}\right)^{*}+V
$$

which contains contribution from triplet of the chiral field $\Phi^{(i)}$.

The potential $V(29)$ is positive by definition (29) and forms a domain wall interpolating between the internal false-vacuum state $(\mathrm{I})\left(V_{(i n t)}=0\right)$ and external gravi-electro-vacuum state (II) $\left(V_{(e x t)}=0\right)$ corresponding to $\mathrm{KN}$ solutions of the Einstein-Maxwell field equations.

The covariant derivations $\mathcal{D}_{\mu}^{(i)}=\nabla_{\mu}+i e A_{\mu}^{(i)}$ contain in general case three different gauge fields $A^{(i)}$. However, in our case we need only one gauge field $A_{\mu}$ associated with the principal chiral field $\Phi^{(1)} \equiv \Phi$. Therefore, we set $F_{\mu \nu}=A_{\mu, \nu}-A_{\nu, \mu}$ and the corresponding covariant derivations will be $\mathcal{D}_{\mu}^{(1)}=\nabla_{\mu}+i e A_{\mu}, \mathcal{D}_{\mu}^{(2)}=\mathcal{D}_{\mu}^{(3)}=\nabla_{\mu}$. the field $\Phi^{(2)} \equiv Z$ is uncharged and $A^{(2)}=0$.

It should be noted that generalization to triplet of the chiral field may be useful for generalization of this model to Salam-Weinberg theory d

\subsection{Problem of the exact solutions.}

We have now to turn to Einstein equations

$$
R_{\mu \nu}-\frac{1}{2} g_{\mu \nu}=8 \pi G T_{\mu \nu}^{(m a t)}
$$

${ }^{\mathrm{c}}(29)$ assumes that $\Phi^{(i)}$ and $\bar{\Phi}^{(i)}$ are independent fields.

$\mathrm{d}$ This idea is suggested by Th.M. Nieuwenhuisen. 
The stress-energy tensor may be decomposed into pure em part and contributions from the chiral fields

$$
\begin{gathered}
T_{\mu \nu}^{(m a t)}=T_{\mu \nu}^{(e m)}+ \\
\delta_{i \bar{j}}\left(\mathcal{D}_{\mu}^{(i)} \Phi^{i}\right) \overline{\left(\mathcal{D}_{\nu}^{(j)} \Phi^{j}\right)}-\frac{1}{2} g_{\mu \nu}\left[\delta_{i \bar{j}}\left(\mathcal{D}_{\lambda}^{(i)} \Phi^{i}\right) \overline{\left(\mathcal{D}^{(j) \lambda} \Phi^{j}\right)}+V\right],
\end{gathered}
$$

and we have to consider three different regions: external zone, $r \geq r_{e}$, transition zone $\left(r_{e}-\delta_{r}\right) \leq r_{e}$, and zone of flat interior $r<\left(r_{e}-\delta_{r}\right)$.

In the external zone, $r \geq r_{e}$, we have $V^{e x t}=0$. The unique nonzero chiral field $\Sigma$ is constant, and therefore, all the derivatives $\mathcal{D}_{\mu}^{(i)} \Phi^{(i)}$ vanish. As a result $T_{\mu \nu}^{(t o t)}$ is reduced to $T_{\mu \nu}^{(e m)}$, and we obtain the Einstein-Maxwell field equations are satisfied and for the external KN electromagnetic field they result in the external KN solution.

For interior of the bubble, $r<\left(r_{e}-\delta_{r}\right)$ we have also $V^{\text {int }}=0$, and the unique nonzero Higgs field is $\Phi(x)=|\Phi(x)| e^{i \chi(x)}$.

The Lagrangian (32) is reduced to (8) with $V(r)=V^{i n t}=0$, which leads to eqs.

$$
\begin{aligned}
\mathcal{D}_{\nu}^{(1)} \mathcal{D}^{(1) \nu} \Phi & =0 \\
\nabla_{\nu} \nabla^{\nu} A_{\mu} & =I_{\mu}=\frac{1}{2} e|\Phi|^{2}\left(\chi_{, \mu}+e A_{\mu}\right) .
\end{aligned}
$$

The only variable chiral field in the flat interior is the oscillating Higgs field, and we have to consider it in more details. One sees that the term

$$
\mathcal{D}_{t}^{(1)} \Phi=\left(\partial_{t}+i e A_{0}^{(i n)}\right)|\Phi| \exp \{i \omega t\}=i\left(\omega+A_{0}^{(i n)}\right) \Phi
$$

is cancelled in agrement with (15), and therefore, (35) is satisfied.

For flat interior the second eq. (36) reduces to the system (13) and we obtain all the consequences considered in sec.5. Therefore, $T_{\mu \nu}^{(m a t)}=0$ and the EinsteinMaxwell equations are trivially satisfied for flat interior.

One sees that considered in sec.5 em solutions together with the discussed here Einstein-Maxwell system, are consistent with the sharp boundary between the external and internal regions, and the limit $\delta_{r} \rightarrow 0$, may be interpreted as a thin wall approximation.

In this limit, external $\mathrm{KN}$ metric matches continuously with flat interior of the bubble and turns out to be consistent with the stress-energy tensor of the external $\mathrm{KN}$ solution and flat interior of the bubble. However, in the thin wall limit, there appears discontinuity in the first derivatives of the metric. Because the Einstein equations contain the second derivatives of the metric, the stress-energy tensor has a $\delta$ - function singularity at the thin wall ${ }^{38}$ In this case of thin wall, the analysis was usually performed on the basis of Israel's formalism of singular layers, ${ }^{13}\left[\frac{38}{3}\right.$ or in terms of generalized functions of the theory of distributions, ${ }^{14}$ The obtained solution may be considered as a consistent with gravity thin wall approximation. However, internal structure of the wall is indeed a non-trivial and very important problem. 


\subsection{Beyond the thin wall approximation.}

Beyond the thin wall approximation, we have to consider as the third zone the zone of phase transition $\left(r_{e}-\delta_{r}\right)<r<r_{e}$.

Zone of phase transition is the practically inaccessible for analytic solutions. Up to our knowledge, analytic solutions of the similar problems with the Higgs field are unknown even for the simple spherical configuration. The known analytic solutions obtained for the vacuum domain walls with planar geometry have a kink-like form, and the typical stress-energy tensor for domain wall has the form

$$
T_{\nu}^{\mu}=\operatorname{diag}(\rho,-\rho,-\rho, 0) .
$$

One expects, that in the case of planar domain wall, the solutions to the full system of the nonlinear equations may in principle be obtained by analytic methods or by the numerical calculations.

Information on the phase transition, produced by the domain wall of the chiral field model, is concentrated in the structure of the stress-energy tensor $T_{\mu \nu}^{(\text {mat })}$, (35) which should be matched with phase transition in gravitational sector in accord to Einstein equations. Gravitational counterpart to this phase transition is the transfer from the flat metric inside the bubble, $r<r_{e}$, to the external metric of the exact $\mathrm{KN}$ solution. For this transfer there is a remarkable ansatz suggested by Gürses and Gürsey (GG) $\stackrel{[39}{\square}$ The standard KS form of the metric (11) with the fixed Kerr congruence $k^{\mu}$, is deformed only in the form of function $H$ (2), which takes the form

$$
H=f(r) / \Sigma, \quad \Sigma=\left(r^{2}+a^{2} \cos ^{2} \theta\right),
$$

where $f$ is arbitrary smooth function.

For the external KN metric $r>r_{e}$, one sets $f(r)=f_{K N}=m r-e^{2} / 2$, while the smooth transfer to some internal metric may be provided by any its smooth extension $f(r)=f_{\text {int }}$. The GG form of metric has remarkable properties. It was shown in $\frac{24}{25}$ that setting for the interior $f(r)=f_{\text {int }}=\alpha r^{4}$, one obtains a regular version of the KN metric, in which the Kerr singularity is suppressed. The GG form of metric describes the rotating solutions with flat asymptotic as well as the rotating versions of the de Sitter and Anti-de Sitter solutions. Moreover, it allows one to match smoothly the external and internal metrics of different sorts. ${ }^{24} 25$ On the other hand the GG form of metrics is a particular case of the Kerr-Schild solutions and inherits its remarkable properties. In particular, the electromagnetic field and its stress-energy tensor display a partial linearization in the Kerr-Schild and GG-spacetimes, and there is the exact correspondence between the rotating and non-rotating solutions which allows one to simplify analysis of the rotating solutions, by means of the analysis of its non-rotating analogs. In particular, the stress-energy tensor of the KN solution and its non-rotating analog with $a=0$ take in the orthonormal tetrad $u, l, n, m$, where $u$ is the unit timelike vector and $l$ the radial one, the form $\frac{16}{24}[25 \mid 39$

$$
T_{\mu \nu}=(8 \pi)^{-1}\left[(D+2 G) g_{\mu \nu}-(D+4 G)\left(l_{\mu} l_{n}-u_{\mu} u_{\nu}\right)\right],
$$


where

$$
G=\frac{f^{\prime} r-f}{\Sigma^{2}}, \quad D=-\frac{f^{\prime \prime}}{\Sigma}
$$

which may be recognized as diagonal one

$$
T_{\nu}^{\mu}=\operatorname{diag}(\rho,-\tau,-\tau, p)
$$

where the radial pressure $p$ and tangential stress $\tau$ are given by

$$
\rho=-p=\frac{1}{8 \pi} 2 G, \quad \tau=-\frac{1}{8 \pi}(D+2 G)=-\rho-\frac{D}{8 \pi} .
$$

This correspondence allows us to consider the phase transfer in the non-rotating case and translate the results to the rotating $\mathrm{KN}$ solution.

For the case of quick rotation, $a>>r_{e}$, there appears a stringy contribution to the mass-energy caused by concentration of the electromagnetic field on the edge of bubble. However, analytic calculations showed (see $\left.{ }^{[17}\right)$ that the 'stringy' contributions from the shell and external em field are mutually cancelled, and the total mass $m_{A D M}^{(\text {total })}=m_{A D M}^{(i n t)}+m_{A D M}^{(\text {shell })}+m_{A D M}^{(e x t)}$ turns out to be equal to $m$. Indeed, this result could be predicted a priori, since the total ADM mass is determined only by the asymptotical gravitational field, i.e. only by the value of parameter $m$ in function $H$, (2) . Therefore, the naive stringy interpretation does not go, at least for the GG form of the metric.

The typical local structure of the stress-energy tensor for the vacuum domain wall $\sqrt[38,40]{4 s}$

$$
T_{\nu}^{\mu}=\rho \operatorname{diag}(1,-1,-1,0),
$$

which shows that the surface energy density $\rho$ is equal to tangential stress $\tau$.

Comparison between (44) and (42) shows that the GG metric contains the domain wall contribution, however there is no full correspondence. It opens a chance to get the the correspondence and stringy effect from some generalizations of the GG form of metric. In particular, the appearance of the string tension may be related with extra dilaton or axion fields, which are typical for the metrics of domain wall models and their analogs in superstring theory ${ }^{42}$ One more very important generalization of this problem may be related with transfer from the used EinsteinMaxwell-Higgs (Chiral) system of the eqs. to its remarkable natural analog in 4D Supergravity $\left[36\right.$ where the complex chiral fields $\phi^{(i)}$ form an extra Kähler manifold supplied by Kähler metric and Kähler potential. It is probably, that the solutions turn out to be consistent with such a generalized system.

We have to stop at this point, since nether analytic calculations for planar domain walls, nor the numerical calculations even for the Einstein-Maxwell-Higgs system were so far performed. There is a great field for activity. 


\section{How the Dirac equation is hidden inside solitonic source of the KN electron.}

As we have shown, the Kerr-Newman solution has many remarkable properties indicating its relationships with the structure of the Dirac electron. However, all these evidences cannot draw us away from the natural question how and where the Dirac equation may be residing in the solitonic source of KN geometry. In this chapter we will try to answer this question. The soliton source of the KN represents a bag confining the Higgs field. In some respects this bag is similar to the MIT and SLAC bag models.

Analyzing the twosheeted structure of the KN solution, we have seen that the Kerr geometry is based on the twistorial structures of the Kerr congruence. The naked Kerr singular ring forms a branch line of space into the sheets of advanced and retarded fields, and the null vector field, $k_{\mu}(x)$ generates the Principal Null Congruence $(\mathrm{PNC}) \mathcal{K}$, form of which is changed by the transfer from positive $(r>0)$ to negative $(r<0)$ sheet. The surface $r=0$ represented a disklike "door" separating two different null congruences $\mathcal{K}^{ \pm}$, creating two different metrics

$$
g_{\mu \nu}^{ \pm}=\eta_{\mu \nu}+2 H k_{\mu}^{ \pm} k_{\nu}^{ \pm}
$$

on the same Minkowski background $M^{4}$.

It seemed that the formation of the soliton source closed this "door" and removed the problem of twosheeted space. However, this problem emerges from another side. The second sheet appeared as a sheet of advanced fields, which are related with the old Dirac problem of radiation reaction. ${ }^{43}$ Dirac splits the expression for retarded potential $A_{r e t}$ into a half-sum and half-difference with advanced fields $A_{a d v}$ as follows

$$
A_{\text {ret }}=\frac{1}{2}\left[A_{\text {ret }}+A_{a d v}\right]+\frac{1}{2}\left[A_{r e t}-A_{a d v}\right] .
$$

The half-sum he takes responsible for self-interaction of the source, while the halfdifference should be responsible for radiation and radiation reaction. The soliton source acquires the second sheet outside the bag as the sheet of advanced fields, which should provide self-interaction of the soliton and create a massive Dirac equation. In accord with the basic properties of the Kerr-Schild solutions, the fields $A_{\text {ret }}$ and $A_{a d v}$ could not reside on the same physical sheet, because each of them should be aligned with the its own Kerr congruence. Considering the retarded sheet as a basic physical sheet, we fix the congruence $\mathcal{K}_{r e t}$ and corresponding metric $g_{\mu \nu}^{+}$, which are not allowed for the advanced field $A_{a d v}$. The advanced field is to be consistent with another congruence $\mathcal{K}_{a d v}$, which should be positioned on a separate sheet with different metric $g_{\mu \nu}^{-}$. However, the problem of their incompatibility disappears inside the bag, where the space is flat, and the both null congruences $\mathcal{K}_{r e t}$ and $\mathcal{K}_{a d v}$ are null not only with respect to the corresponding Kerr-Schild metrics, but also with respect to the flat Minkowski background.

For the sake of convenience, we replace further notations $\mathcal{K}_{\text {ret }}$ and $\mathcal{K}_{a d v}$ by the notations $\mathcal{K}^{ \pm}$, identifying $\mathcal{K}^{+}=\mathcal{K}_{\text {ret }}, \mathcal{K}^{-}=\mathcal{K}_{a d v}$. The external null fields $k_{\mu}^{ \pm}(x)$ 


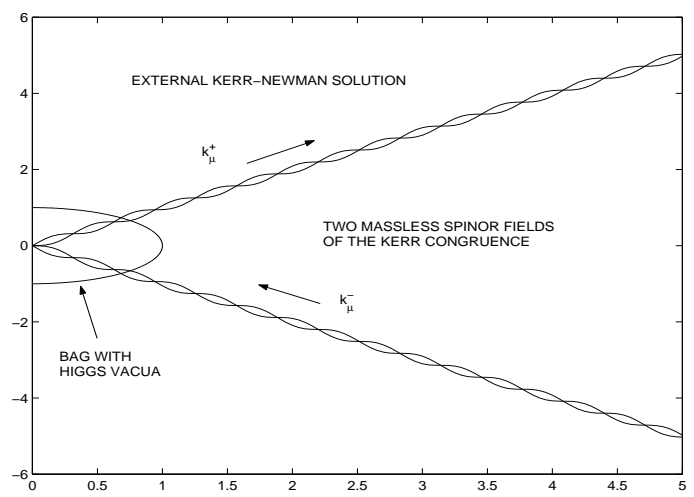

Fig. 4. Creation of the Dirac equation inside the bag by the confined Higgs field coupled to two massless spinor fields $k_{\mu}^{+}$and $k_{\mu}^{-}$, generators of the Kerr principal null congruences $\mathcal{K}^{+}$and $\mathcal{K}^{-}$.

are extended inside the bag on the same sheet forming two different null congruences determined by two conjugate solutions of the Kerr theorem $Y^{ \pm}\left(x^{\mu}\right), 11,45$

Now, we have to say a few words on the Kerr theorem. The Kerr theorem is formulated in the Minkowski background, $x^{\mu}=(t, x, y, z) \in M^{4}$, in terms of the projective twistor coordinates

$$
T^{A}=\{Y, \zeta-Y v, u+Y \bar{\zeta}\}, \quad A=1,2,3,
$$

where $u, v, \zeta, \bar{\zeta}$ are the null Cartesian coordinates, $\zeta=(x+i y) / \sqrt{2}, \quad \bar{\zeta}=(x-$ iy) $/ \sqrt{2}, u=(z+t) / \sqrt{2}, \quad v=(z-t) / \sqrt{2}$. The Kerr congruence is determined by solution of the equation

$$
F\left(T^{A}\right)=0,
$$

where $F$ in general case is a holomorphic function on the projective twistor space $C P^{3}$. For the Kerr solution the congruence is created by function $F$ which is quadratic in $Y$ and may be represented in the form

$$
F\left(Y, x^{\mu}\right)=A\left(x^{\mu}\right) Y^{2}+B\left(x^{\mu}\right) Y+C\left(x^{\mu}\right), .
$$

In this case the equation (48) has two explicit solutions

$$
Y^{ \pm}\left(x^{\mu}\right)=(-B \mp \tilde{r}) / 2 A,
$$

where $\tilde{r}=\left(B^{2}-4 A C\right)^{1 / 2}$, and it was shown in, $\frac{47}{4}$ that these two solutions are related by antipodal correspondence

$$
Y^{+}=-1 / \bar{Y}^{-} .
$$

It should be noted, that $Y$ is a projective spinor coordinate, $Y=\phi_{1} / \phi_{0}$, and it is equivalent to the Weyl two-component spinor $\phi_{\alpha}=\left(\phi_{1}, \phi_{0}\right)^{T}$.

In the Kerr-Schild formalism, $\frac{11}{11}$ function $Y\left(x^{\mu}\right)$ determines the null congruence as a field of null directions $k_{\mu}\left(x^{\mu}\right)$ via differential form

$$
k_{\mu} d x^{\mu}=P^{-1}(d u+\bar{Y} d \zeta+Y d \bar{\zeta}-Y \bar{Y} d v)
$$


where $P=(1+Y \bar{Y}) / \sqrt{2}$ is a normalizing factor. This form is equivalent to standard representation of the null vector via spinor $\phi$ and Pauli matrices $k_{\mu}^{+}=\bar{\phi} \bar{\sigma}_{\mu} \phi$, and therefore, generating two conjugate null congruences, the Kerr theorem determines simultaneously two massless spinor fields of different chirality $\phi_{\alpha}=\left(\phi_{1}, \phi_{0}\right)^{T}$ and $\chi^{\dot{\alpha}}=\left(\chi^{\dot{1}}, \chi^{\dot{0}}\right)^{T}$

We can compare these spinor fields with the structure of the Dirac equation

$$
\left(\gamma^{\mu} \hat{\Pi}_{\mu}+m\right) \Psi=0
$$

in which $\Psi=\left(\phi_{\alpha}, \chi^{\dot{\alpha}}\right)^{T}$ and $\hat{\Pi}_{\mu}=-i \partial_{\mu}-e A_{\mu}$, and $\hat{\Pi}_{\mu}=-i \partial_{\mu}-e A_{\mu}$, written in the Weyl basis, where it splits in two equations ${ }^{46}$

$$
\bar{\sigma}^{\mu \dot{\alpha} \alpha}\left(i \partial_{\mu}+e A_{\mu}\right) \phi_{\alpha}=m \chi^{\dot{\alpha}}, \quad \sigma_{\alpha \dot{\alpha}}^{\mu}\left(i \partial_{\mu}+e A_{\mu}\right) \chi^{\dot{\alpha}}=m \phi_{\alpha} .
$$

In the Standard Model these equations are called the "left handed" and the "right handed electron fields". In the massless case (54) represents two equations, for two Weyl spinors, connected by antipodal relation (51). The corresponding null vectors

$$
k_{L}^{\mu}=\bar{\phi} \sigma^{\mu} \phi, \quad k_{R}^{\mu}=\bar{\chi} \bar{\sigma}^{\mu} \chi, \quad k_{\mu L} k_{L}^{\mu}=k_{\mu R} k_{R}^{\mu}=0,
$$

describe two principal null congruences given by antipodal solutions $Y^{ \pm}$of the Kerr theorem. Outside the bag these null fields should reside on different sheets of the KN solution, but penetrating inside the bag they are meeting without conflict and can reside on the same false-vacuum sheet because of flatness of the space inside the bag. bag. Outside the bag they form a four-component Dirac spinor $\Psi(x)$ satisfying the massless Dirac equation

$$
\left(\gamma^{\mu} \hat{\Pi}_{\mu}\right) \Psi=0,
$$

which acquires mass term inside the bag from the Higgs field $\Phi$ through the Yukawa interaction

$$
\mathcal{L}_{\text {Yukawa }}(\Phi, \Psi)=-g \bar{\Psi} \Phi \Psi .
$$

Therefore, two antipodal twistorial congruences, obtained as two conjugate solutions of the Kerr theorem $Y^{ \pm}(x)$, create two massless spinor fields $\phi_{\alpha}$ and $\chi^{\dot{\alpha}}$, which are combined in a four-component massless Dirac field $\Psi=\left(\phi_{\alpha}, \chi^{\dot{\alpha}}\right)^{T}$. The Higgs field $\Phi$ confined inside the bag connects them by the Yukawa coupling (57), which gives the mass term to the Dirac equation in full agreement with the one of principal tasks of the Higgs field in the Standard Model.

\section{Outlook: relation to superstring theory.}

Solitonic regularization The negative sheet of the metric disappears and metric turns out to be regularized and practically flat. The model contains the lightlike heterotic string on the border of the bubble, however, it is axially symmetric and the traveling waves are absent. Therefore, some extra excitations are needed to create the traveling waves. 
Does the KN model of electron contradict to Quantum Theory? It seems "yes", if one speaks on the "bare" electron. However, in accordance with QED, vacuum polarization creates in the Compton region a cloud of virtual particles forming a "dressed" electron. This region gives contribution to electron spin, and performs a procedure of renormalization, which determines physical values of the electron charge and mass. We describe here an alternative solitonic version of the regularization performed on the base of the Higgs field, which seems to us a more physical model contrary to the used in QED formal mathematical model.

Speaking on the "dressed" electron, one can say that the physical contradiction between the KN model and the Quantum electron in QED is absent. Note however, that dynamics of the virtual particles in QED is chaotic and can be conventionally separated from the "bare" electron. In the same time, the vacuum state inside the Kerr-Newman soliton forms a coherent oscillating state joined with a closed Kerr string. The oscillating bubble source represents an integral whole with the extended electron, and its 'internal' structure cannot be separated from a "bare" particle.

The KN electromagnetic field is regularized since the maximal value of the vector potential is realized in the equatorial plane, on the stringy boundary of the bubble. Note, that radius of the regularized closed string, being shifted to the boundary of the bubble, turns out to be slightly increased. The position of the string confirms the known suggestion by Witten that the heterotic strings have to be formed on the boundary of a domain wall.

It should be noted that the real closed Kerr string is only the peak of the iceberg. As it was shown in the recent paper ${ }^{8}$ there is deep parallelism between the complex structure of the Kerr geometry and basic structures of the superstring/M-theory. In particular, about two decades ago, author obtained a complex string inside of the complex 4D Kerr geometry, which together with the closed heterotic string of the KN source forms a membrane source of the M-theory. The closed heterotic Kerr string is light-like, and its structure is similar to the Sen fundamental string solution to low-energy heterotic string theory. In the recent paper ${ }^{48}$ Adamo and Newman reobtained these two strings analyzing asymptotic form of the geodesic and shearfree congruences. Their emotionally comments are worth quoting: "...It would have been a cruel god to have layed down such a pretty scheme and not have it mean something deep."

In the recent papers, $\frac{8}{47}$ there was obtained a new remarkable fact - appearance of the Calabi-Yau twofold (K3 surface) in the projective twistor space $C P^{3}$. Therefore, the famous K3 surface of the superstring theory represents nothing other than a twistorial description of the Principal Null Congruence (PNC) of the Kerr geometry. One can suppose, that this parallelism is not accidental, and there should be an underlying superstring structure lying beyond these relationships, and we suggest that it is $\mathrm{N}=2$ critical superstring. The $\mathrm{N}=2$ superstring is one of the three string theories consistent with quantum theory; number of supersymmetries $\mathrm{N}=0$, 1, 2 corresponds to consistent space-time dimensions $\mathrm{D}=26, \mathrm{D}=10$ and $\mathrm{D}=4,{ }^{7}$ The $\mathrm{N}=2$ string, having complex dimension two, was very popular a few decades ago ${ }^{7}$ 
However, it has unusual signature which is conflicted with the real minkowskian space-time. As a result, it was almost forgotten, and in particular it does not discussed in the very good modern textbook $\frac{49}{[1}$ It has been shown recently in, $\stackrel{8}{ }$ that the $\mathrm{N}=2$ string can be embedded in the complex Kerr geometry, and moreover, there are evidences that it can be considered as a complex source of the Kerr geometry.

\section{Acknowledgments}

This work is supported under the RFBR grant 13-01-00602. Author thanks Theo M. Nieuwenhuizen for permanent interest to this work and useful conversations.

\section{References}

\section{References}

1. Schwarz J H, The Early Hystory of String Theory and Supersymmetry, arXiv:1201.0981.

2. F. Wilczek, The Lightness Of Being (Basic Books, 2008).

3. L. Susskind, The Black Hole War (Hachette Book Group US, 2008).

4. B. Carter, Phys. Rev. 174, 1559 (1968).

5. A. Sen Macroscopic Charged Heterotic String Nucl.Phys. B 388457 (1992) arXiv:hep-th/9206016.

6. J. Bjorken and S. Drell, Relativistic Quantum Mechanics (McGraw Hill Book, 1964).

7. M.B. Green, J. Schwarz, and E. Witten, Superstring Theory, v.I, (Cambridge Univ. Press, 1987).

8. A. Burinskii, Adv.High Energy Phys. 2013:509749, Doi:10.1155/2013/509749, arXiv:1211.6021.

9. D.D. Ivanenko and A.Ya. Burinskii, Izv. Vuz. Fiz., 5, 135 (1975).

10. E. T. Newman et al., J. Math. Phys., 6, 918 (1965).

11. G. C. Debney, R. P. Kerr and A. Schild, J. Math. Phys. 10, 1842 (1969).

12. A.Ya. Burinskii, Soviet Phys. JETP 39, 193 (1974).

13. W. Israel, Phys. Rev. D 2, 641 (1970).

14. C.A. López, Phys. Rev. D, 30 (1984) 313.

15. A. Burinskii, Grav. Cosmol. 14, 109 (2008), arXiv:hep-th/0507109].

16. I. Dymnikova, Phys. Lett. B 639, 368 (2006).

17. A. Burinskii, J. Phys. A: Math. Theor. 43 (2010) 392001 [arXiv: 1003.2928].

18. Th.M. Nieuwenhuizen, The Electron and the Neutrino as Solitons in Classical Electrodynamics, In: Beyond the Quantum, eds. Th.M. Nieuwenhuizen et al., World Scientific, Singapure, 2007), pp.332-342.

19. A. Burinskii, J. of Phys.: Conf. Series, 361, 012032 (2012), arXiv:1109.3872.

20. A. Burinskii, J, of Physics: Conference Series, 343, 012019 (2012), arXiv:1112.0225.

21. A. Sen, Rotating charged black hole solution in heterotic string theory, Phys. Rev. Lett. 691006 (1992).

22. H. Keres, On physical interpretation of solution of the Einstein equations, Soviet Phys. JETP 25, (1967) 504.

23. V. Hamity, Phys. Lett. A 5677 (1976).

24. A. Burinskii, Supersymmetric Superconducting Bag as a Core of Kerr Spinning Particle, Grav. Cosmol. 8, 261 (2002), arXiv:hep-th/0110011.

25. A. Burinskii, E. Elizalde, S.R. Hildebrandt and G. Magli Phys. Rev. D 65, 064039 (2002), arXiv:gr-qc/0109085 
26. D. Garfinkle, Phys. Rev. D 464286 (1992).

27. A. Dabholkar, J. P. Gauntlett, J. A. Harvey, D. Waldram Nucl.Phys. B 474 85-121 (1996), arXiv:hep-th/9511053,

28. A. Burinskii, Some properties of the Kerr solution to low-energy string theory, Phys. Rev. D 52 (1995) 5826, arXiv:hep-th/9504139.

29. H. B. Nielsen and P. Olesen, Nucl. Phys. B 61, 45 (1973).

30. G. Rosen, Particlelike solutions to nonlinear complex scalar field theories with positive definite energy densities, J. Math. Phys. 9, 996 (1968).

31. S. Coleman, Q-Balls, Nuclear Physics B 262 (2) 263 (1985).

32. M.Volkov and E. Wöhnert, Phys.Rev. D 66, 085003 (2002).

33. N.Graham, Phys.Rev.Lett 98, 101801 (2007).

34. F.E.Schunck and E.W.Mielke, in Relativity and Scientific Computing, edited by F.Hehl at.al., Springer, Berlin, 1966, pp.138-151. Sh.Yoshida and Yo.Eriguchi, Phys.Rev. D56, 762(1997);

35. E. Witten, Nucl. Phys. B 249, 557 (1985).

36. Wess J and Bagger J 1983 Supersymmetry and Supergravity (Princeton Univ. Press, Princeton, New Jersey)

37. J. R. Morris, Phys. Rev. D 53, 2078 (1996) arXiv:hep-ph/9511293.

38. M. Cvetič, S. Griffies, and H. H. Soleng, Phys. Rev. D 48, 2613 (1993).

39. M. Gürses and F. Gürsey, J. Math. Phys. 16, 2385 (1975).

40. J.Ipser and P. Sikive, Phys. Rev. D 30, 712 (1984).

41. M. Cvetič, S. Griffies, and S. Rey, Nucl. Phys. B 381, 301 (1992).

42. M. Cvetič, F. Quevedo and S.-J. Rey, Phys. Rev. Lett. 67, 1836 (1991).

43. P. A. M. Dirac, Clasical theory of radiating electrons, Proc. R. Soc. London, Ser. A 167, 148 (1938).

44. V.B. Berestetsky, E.M. Lifshitz, L.P. Pitaevsky, Quantum Electrodynamics ( Course Of Theoretical Physics, 4), Oxford, Uk: Pergamon ( 1982).

45. D. Kramer, H. Stephani, E. Herlt, M. MacCallum, Exact Solutions of Einstein's Field Equations, Cambridge Univ. Press, Cambridge (1980).

46. V. B. Berestetsky, E. M. Lifshitz, L. P. Pitaevsky, Quantum Electrodynamics (Course Of Theoretical Physics, 4), Oxford, Uk: Pergamon (1982).

47. A. Burinskii, Theor. Math. Phys., 177(2), 1492 - 1504, (2013).

48. T.M. Adamo and E.T. Newman, Light cones in relativity: Real, complex and virtual, with applications, Phys. Rev. D 83 (2011) 044023

49. K. Becker, M. Becker and J. Schwarz, String Theory and M-Theory - A Modern Introduction, Cambridge University Press, 2007. 